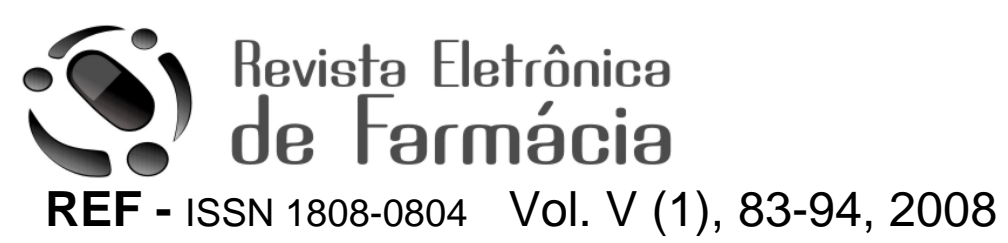

\title{
APLICABILIDADE DE DOIS INSTRUMENTOS DE AVALIAÇÃO DE SERVIÇOS EM FARMÁCIA HOSPITALAR
}

\author{
Applicability Of Two Evaluation Instruments Of Services In Hospital Pharmacy \\ Bárbara Posse Reis Martins; ${ }^{1}$ Camile Vechiato; ${ }^{1}$ Daniela Alves Vieira; ${ }^{1}$ Daniela Mendes Borges; ${ }^{1}$ Mércia \\ Pandolfo Provin ${ }^{2}$ \\ ${ }^{1}$ Farmacêuticas especialistas em farmácia hospitalar e assistencial pela Faculdade de Farmácia / Universidade \\ Federal de Goiás. \\ ${ }^{2}$ Professora da Disciplina de Farmácia Hospitalar da FF/UFG
}

Autor para correspondência e-mail: barbaraposse@yahoo.com.br

Recebido em 16/01/2008 - Aceito em 17/07/2008

RESUMO: A avaliação de uma farmácia hospitalar é uma prática complexa e difícil por envolver estruturas e serviços variáveis de acordo com as características do hospital e da própria farmácia. Este trabalho teve como objetivo analisar a aplicabilidade e os resultados de dois instrumentos de avaliação de serviços em farmácia hospitalar, são eles: o guia da Sociedade Espanhola de Farmácia Hospitalar (SEFH) e um segundo guia proposto pelas pesquisadoras. Foi aplicado roteiro de investigação proposto pelos dois guias em 12 hospitais da cidade de Goiânia. Em ambos os instrumentos a avaliação consistiu na atribuição de pontos para as atividades desenvolvidas pelos serviços de farmácia. As pontuações finais obtidas foram divergentes entre os dois guias. $\mathrm{O}$ instrumento da SEFH se mostrou mais sensível e seguro, no entanto é muito complexo, exigindo bastante tempo para sua aplicação e análise. O instrumento proposto é mais simples e rápido, no entanto apenas fornece informações superficiais sobre os serviços não os avaliando profundamente. Os resultados encontrados demonstram a necessidade da elaboração de um instrumento oficial que avalie de maneira quali e quantitativa os serviços de farmácia hospitalar, que possam dar suporte as tomadas de decisão dos gestores desses serviços, visando melhoras e adequações. A aplicação e análise dos dois guias permitiram identificar e analisar as principais atividades e os problemas dos serviços de farmácia destes hospitais.

PALAVRAS-CHAVE: Avaliação. Instrumento de avaliação. Farmácia hospitalar.

ABSTRACT: The evaluation of a hospital pharmacy is difficult and complex because it involves changeable structures and services in accord with the pharmacy and with characteristics related to the hospital. This study had as an aim the applicability and the results of two evaluation instruments of hospital pharmacy services, they are: the guide of the Spanish Society of Pharmacy Hospital (SEFH) and the guide proposed by the researchers. It was applyed the two guides in twelve hospitals in Goiânia. In both of the instruments, the evaluation consisted in giving points for the activities developed for the pharmacy services. The punctuations were divergent between the two guides. The instrument of the SEFH revealed more sensible and safety, however it is very complex, demanding sufficiently time for its application and analysis. The other instrument is simpler and fast, however it only supplies superficial information on the services, not evaluate them deeply. The joined results demonstrate the necessity of the creation of an official instrument that evaluates in quality and quantity of way the services that can give support for the taking decisions of managers of these services aiming at improvements and adaptations. The application and analysis of the two guides had allowed to identify and analyze to the main activities and the problems of the pharmacy services of these hospitals.

KEY WORDS: Evaluation. Evaluation instrument. Hospital pharmacy. 
Martins, B. P. R. et al./Revista Eletrônica de Farmácia Vol 5(1), 83-94, 2008.

Farmácia Hospitalar é conceituada pela Sociedade Brasileira de Farmácia Hospitalar (SBRAFH) como "unidade clínica, administrativa e econômica, dirigida por profissional farmacêutico, ligada hierarquicamente à direção técnica do hospital e integrada funcionalmente às demais unidades de assistência ao paciente".

É um setor do hospital extremamente importante, dentre suas principais atribuições destacam-se: participação nas comissões intra-hospitalares (Comissão de Farmácia e Terapêutica, Comissão de Controle de Infecção Hospitalar, Suporte Nutricional, Compras e Licitações, e outras que podem ser constituídas), farmacotécnica, farmácia clínica, aquisição, armazenamento, distribuição e gestão de estoque, promoção do uso racional de medicamentos, farmacovigilância e monitoramento de reações adversas a medicamentos, seleção de medicamentos, atenção farmacêutica, implantação do Centro ou Sistema de Informações de Medicamentos além de outras atividades que garantem a qualidade da assistência ao paciente (SBRAFH, 1996; GOMES \& REIS, 2003).

No contexto atual, o farmacêutico hospitalar deve assumir um perfil clínico-assistencial, atuando em todas as fases da terapia medicamentosa, cuidando, em cada momento, de sua adequada utilização nos planos assistencial, econômico, de ensino e de pesquisa (GOMES \& REIS, 2003).

O papel conquistado pelo farmacêutico no cuidado do paciente tem sido cada vez mais relevante. Suas primeiras atuações surgiram como conseqüência da implantação de sistemas racionais de distribuição de medicamentos, os quais permitiram um conhecimento completo da medicação que os pacientes recebiam. (CASTRO et al., 2000).

A Resolução $n^{\circ}$ 300, de 30/01/1997 do Conselho Federal de Farmácia (BRASIL, 1997a) regulamenta o exercício profissional em farmácia hospitalar e define suas competências. A Agência Nacional de Vigilância Sanitária (ANVISA) regulamenta e estabelece as diretrizes para o funcionamento deste setor através de resoluções e portarias.

Todas as legislações aplicadas à farmácia hospitalar estabelecem os requisitos mínimos para o adequado funcionamento do setor e são a base das ações de fiscalização sanitária que verificam o cumprimento às exigências legais.

A acreditação é um sistema internacional cujo objetivo é a promoção da constante avaliação e certificação da qualidade dos serviços de saúde, garantindo assim a qualidade na assistência em todas as organizações prestadoras de serviços de saúde do País. O Sistema Nacional de Acreditação é coordenado, no Brasil, por uma entidade não governamental, a Organização Nacional de Acreditação (ONA), que possui caráter educativo e não fiscalizador. Suas atividades estão voltadas para o treinamento e capacitação, elaboração de manuais e avaliação dos recursos institucionais para certificação. A acreditação dos serviços de saúde é uma ferramenta para a qualificação e melhora dos serviços de saúde sendo reconhecido oficialmente pela ANVISA através da resolução no 921de 29 de maio de 2002 (BRASIL, 2002b ; ONA, 2007).

A Sociedade Brasileira de Farmácia Hospitalar define os padrões mínimos para esses serviços, a Organização Nacional de Acreditação define a avaliação para certificação dos serviços de saúde, o Conselho Federal de Farmácia e a Agência Nacional de Vigilância Sanitária regulamentam esses serviços, no entanto, não há disponível no Brasil um instrumento adequado à avaliação qualitativa e quantitativa dos serviços de farmácia hospitalar.

Segundo Donabedian, o enfoque da avaliação de serviços de saúde pode ser o profissional de saúde ou a instituição e o processo avaliativo deve analisar estrutura, processo e resultados (DONABEDIAN, 1988).

Desde 1992 entidades brasileiras revelam a necessidade e importância de instrumentos que avaliem os serviços de farmácia hospitalar no Brasil (MAGARINOS-TORRES, OSORIO-DE-CASTRO \& PEPE, 2007).

A Sociedade Espanhola de Farmácia Hospitalar elaborou e publicou um instrumento com essa finalidade, porém, a utilização desse instrumento nos serviços brasileiros ainda precisa ser testada e validada.

A realização deste trabalho teve como objetivo comparar a aplicabilidade de dois instrumentos de avaliação de serviços de farmácias hospitalares. O primeiro deles proposto pelas autoras e o segundo pela Sociedade Espanhola de Farmacia Hospitalaria - SEFH, 2007.

\section{METODOLOGIA}

Dois instrumentos distintos para avaliação de serviços de farmácia hospitalar foram aplicados em unidades hospitalares públicas e privadas na cidade de Goiânia, Goiás em agosto de 2007.

\section{Unidades Hospitalares}

As farmácias hospitalares foram identificadas a partir das listas de hospitais fornecidas pelo Departamento de Vigilância Sanitária Municipal de Goiânia e pelo Conselho Regional de Medicina do Estado de Goiás, totalizando um universo de 73 hospitais.

Os hospitais foram distribuídos em 3 subgrupos de acordo com o regime jurídico e o porte (Quadro 1), foram excluídos os hospitais psiquiátricos, aqueles cujo tempo de internação era inferior a 24 horas e aqueles que se recusaram a participar do estudo. 
Martins, B. P. R. et al./Revista Eletrônica de Farmácia Vol 5(1), 83-94, 2008.

Quadro 1: Subgrupos de hospitais de acordo com o porte.

\begin{tabular}{cc}
\hline Número de Leitos & Porte do Hospital \\
\hline Menos que 50 & Pequeno \\
50 a 200 & Médio \\
Acima de 200 & Grande \\
\hline
\end{tabular}

Para compor amostra, foram selecionados de maneira randômica 3 hospitais de cada subgrupo, exceto para o subgrupo público de porte médio e privado de grande porte onde foram identificados respectivamente 2 e 1 instituições de cada na cidade. Um hospital de privado de pequeno porte, um público de médio e um de grande porte se recusaram a participar do estudo (Quadro 2).

Quadro 2: Composição dos subgrupos de hospitais estudados.

\begin{tabular}{lcc}
\hline \multicolumn{1}{c}{ Hospitais pesquisados } & Quantidade & Denominação \\
\hline Privado de pequeno porte & 02 & PPP1; PPP2 \\
Privado de médio porte & 03 & PMP1; PMP2; \\
& & PMP3 \\
Privado de grande porte & 01 & PGP1 \\
Público de pequeno porte & 03 & PUPP1; PUPP2; \\
& & PUPP3 \\
Público de médio porte & 01 & PUMP1 \\
Público de grande porte & 02 & PUGP1; PUGP2
\end{tabular}

\section{Instrumentos}

O Guia de avaliação e melhora dos serviços de farmácias hospitalares desenvolvido pela Sociedade Espanhola de Farmácia Hospitalar - SEFH (2007) foi um dos instrumentos escolhidos para avaliar sua aplicabilidade em serviços de farmácia em hospitais de Goiânia e está disponível no site da Sociedade Espanhola de Farmácia Hospitalaria.

O instrumento da SEFH classifica os serviços em 4 patamares (Quadro 3), por meio da pontuação final obtida na avaliação, cujo valor máximo é 100. O guia abrange as atividades: gestão, distribuição, farmacotécnica, farmacocinética, farmacovigilância, toxicologia e unidade de radiofarmácia. Cada atividade tem um peso específico e valor máximo de 100 pontos.

As atividades podem se dividir em áreas e subáreas as quais são atribuídas pontos segundo a sua relevância. Todas essas áreas e subáreas se classificam em três níveis: III, II e I em ordem crescente de complexidade. Cada nível está definido por uma série de itens que estão agrupados por categorias A, B e C que visam representar sempre que possível a implantação, a atividade e a qualidade do serviço. Em algumas situações as três categorias encontram-se agrupadas em um só item. A pontuação final de cada nível é obtida pela soma dos itens, sendo necessário atingir uma pontuação mínima equivalente a $50 \%$ do valor total do nível, para poder passar para o próximo nível, ou seja, para passar para o nível I (mais complexo) é necessário ter cumprido todos os itens do nível III (menos complexo). A pontuação final de cada atividade é a soma dos pontos obtidos em cada área.

Para o cálculo da pontuação final da avaliação é realizado a somatória dos pontos das sete atividades, sendo aplicado o fator de peso específico para cada uma.

Quadro 3: Classificação dos serviços de farmácia pelo guia da SEFH, em 4 patamares definidos, de acordo com a pontuação final obtida

\begin{tabular}{cc}
\hline Patamar & Pontuação \\
\hline I & Mais de 75 \\
II & $55-74$ \\
III & $35-54$ \\
IV & Menos de 35 \\
\hline
\end{tabular}

O instrumento elaborado pelas autoras foi adaptado a partir do instrumento utilizado por Osório-de-Castro (2004), nele a pontuação final é de 100 pontos divididos, segundo sua relevância, definidos a critério das autoras, nas seguintes atividades: gestão, farmacovigilância, distribuição, farmacotécnica e ensino e pesquisa. 
Martins, B. P. R. et al./Revista Eletrônica de Farmácia Vol 5(1), 83-94, 2008.

O preenchimento dos instrumentos de avaliação foi realizado pelas autoras mediante entrevista com os farmacêuticos ou outro responsável pelas farmácias hospitalares.

Os dados coletados foram consolidados nos próprios instrumentos e posteriormente digitados, para análise quantitativa e qualitativa.

\section{RESULTADOS E DISCUSSÃO}

A SEFH criou seu instrumento de avaliação com a finalidade de analisar os serviços de farmácias hospitalares visando melhora na sua atividade e qualidade. Sua elaboração se deu com a colaboração de vários especialistas e foi testado e validado (SEFH, 2007).

Trata-se, entretanto, de uma avaliação complexa e extensa que contempla todas as atividades que podem ser desenvolvidas pelo serviço de farmácia, em diferentes níveis e situações, sendo que muitos desses serviços não são realizados pelas farmácias hospitalares brasileiras.

Suas desvantagens consistem no tempo dispendioso para sua aplicação e análise e na dificuldade de interpretação. O mesmo não faz referência nem avalia a estrutura física dos serviços de farmácia, sendo mais uma desvantagem, pois estrutura física adequada e de acordo com a legislação sanitária é imprescindível para o bom funcionamento do serviço.

O instrumento proposto é de aplicação mais fácil e rápida e aborda somente as atividades comumente desenvolvidas pelas farmácias hospitalares do Brasil. Mas a maior praticidade e facilidade não refletem em resultados muito seguros, pois o guia não avalia tão profundamente quanto o guia da SEFH. As características dos dois guias podem ser verificadas no Quadro 4.

Quadro 4: Comparação entre os guias de avaliação

\begin{tabular}{lcc}
\hline \multicolumn{1}{c}{ Característica } & SEFH & PROPOSTO \\
\hline Ambigüidade & médio & pouco \\
Análise dos resultados & muito & pouco \\
Atividades abordadas & muito & médio \\
Avaliação da qualidade & muito & pouco \\
Complexidade & muito & pouco \\
Confiabilidade & muito & pouco \\
Dificuldade de aplicação & muito & pouco \\
Efetividade & médio & médio \\
Eficácia & muito & pouco \\
Entendimento & pouco & muito \\
Reprodutibilidade & muito & muito \\
Sensibilidade & muito & pouco \\
Tamanho & muito & médio \\
Tempo gasto para aplicação e análise & muito & médio \\
\hline
\end{tabular}

A análise dos resultados encontrados demonstra que o guia da SEFH, embora não adaptado para a realidade brasileira, é mais abrangente que o guia proposto.

Os dois guias foram eficientes para identificar as não conformidades dos serviços visitados.

A discrepância entre os resultados finais (Tabela 1; Gráfico 1), revelam a sensibilidade e especificidade do instrumento espanhol. A diferença média entre os resultados obtidos pelos dois instrumentos foi de aproximadamente 35 , sendo que as menores pontuações foram apontadas no guia espanhol.

O desvio padrão das pontuações das amostras avaliadas pelo guia da SEFH foi de 13,6 e o das amostras avaliadas pelo guia proposto foi de 17,9. O menor desvio padrão observado no guia da SEFH demonstra que o mesmo avalia os hospitais de maneira mais homogênea do que o guia proposto, sendo deste modo, mais seguro e eficaz.

Tabela 1: Pontuação final obtida pelos hospitais avaliados, por guia de avaliação.

\begin{tabular}{cccc}
\hline HOSPITAL & SEFH & PROPOSTO & VARIAÇÃO \\
\hline PPP1 & 6,7 & 33,8 & 27,1 \\
PPP2 & 20,6 & 36,8 & 16,2 \\
PMP1 & 13,9 & 43,9 & 30,0 \\
PMP2 & 20,2 & 56,2 & 36,0 \\
PMP3 & 47,6 & 97,5 & 49,9 \\
PGP1 & 51,0 & 78,8 & 27,8 \\
PUPP1 & 17,7 & 51,8 & 34,1
\end{tabular}


Martins, B. P. R. et al./Revista Eletrônica de Farmácia Vol 5(1), 83-94, 2008.

\begin{tabular}{cccc} 
PUPP2 & 15,0 & 51,4 & 36,4 \\
PUPP3 & 10,3 & 53,4 & 43,0 \\
PUMP1 & 21,5 & 54,7 & 33,2 \\
PUGP1 & 20,9 & 70,0 & 49,2 \\
PUGP2 & 27,1 & 62,5 & 35,4 \\
Média & 22,7 & 57,7 & 34,9 \\
Desvio padrão & 13,6 & 17,9 & - \\
\hline
\end{tabular}

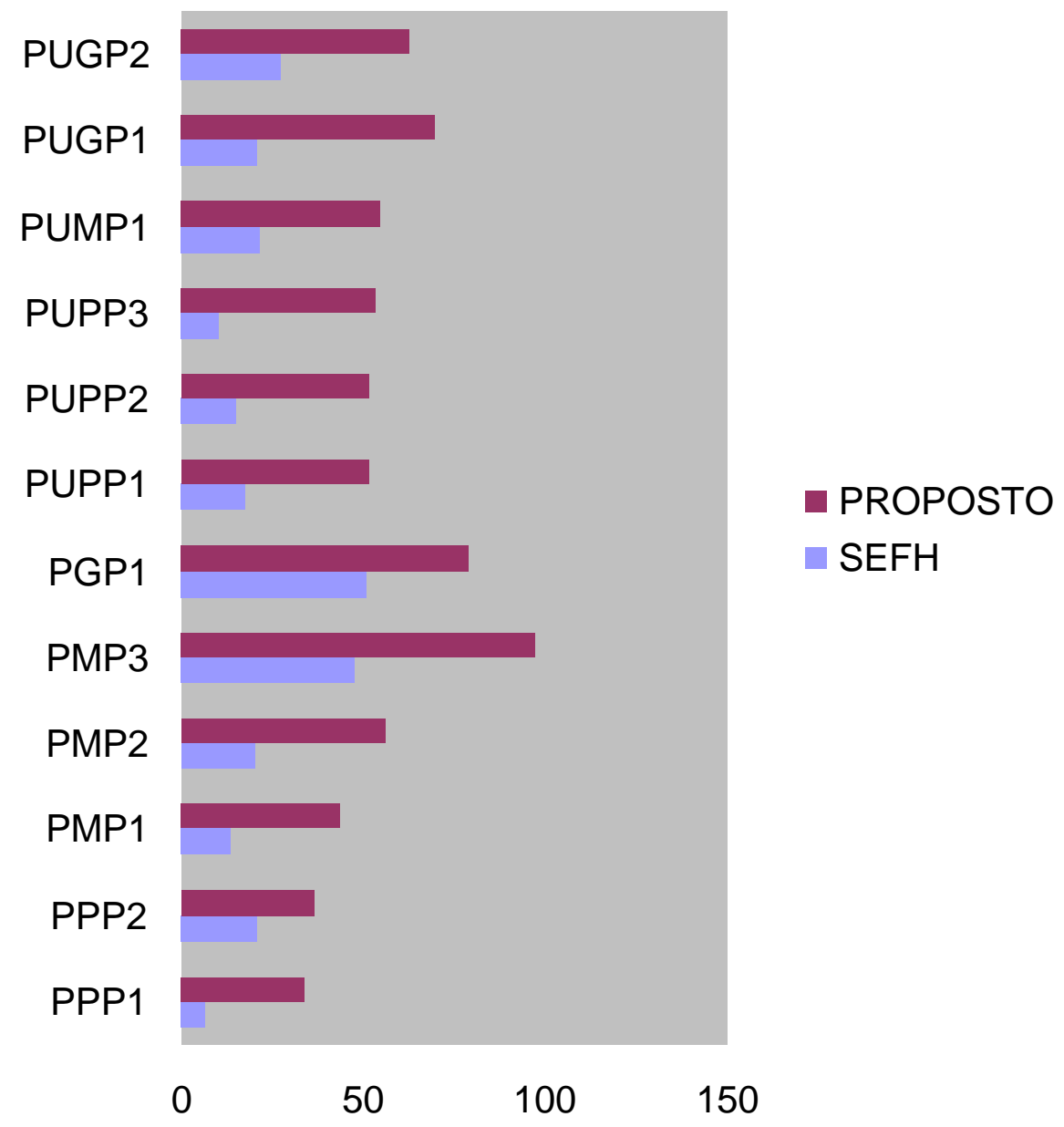

Gráfico 1: Pontuação final obtida pelos hospitais nos dois guias de avaliação.

O guia de avaliação da SEFH, classifica os serviços de farmácia em 4 patamares, decrescentes, sendo o patamar I o mais alto e qualificado serviço e o patamar IV o mais baixo. Dos 12 hospitais avaliados pelo guia da SEFH, 2 (16,7\%) foram classificados no patamar III, com uma média de pontos equivalente a 49,3 e 10 (83,3\%) no patamar IV, com uma média de pontos equivalente a 17,4. Nenhuma das farmácias hospitalares avaliadas obteve pontuação para ser classificada nos patamares II e I. A pontuação mínima obtida foi de 6,7 e a máxima de 51,0 (Tabela 2 e 3).

Tabela 2: Pontuação e patamar de classificação obtido pelos serviços de farmácias avaliadas, de acordo com o guia da SEFH.

\begin{tabular}{ccc}
\hline Hospital & Pontuação & Patamar de classificação \\
\hline PPP1 & 6,7 & IV \\
PPP2 & 20,6 & IV \\
PMP1 & 13,9 & IV \\
PMP2 & 20,2 & IV
\end{tabular}


Martins, B. P. R. et al./Revista Eletrônica de Farmácia Vol 5(1), 83-94, 2008.

\begin{tabular}{cll} 
PMP3 & 47,6 & III \\
PGP1 & 51,0 & III \\
PUPP1 & 17,7 & IV \\
PUPP2 & 15,0 & IV \\
PUPP3 & 10,3 & IV \\
PUMP1 & 21,5 & IV \\
PUGP1 & 20,9 & IV \\
PUGP2 & 27,1 & IV \\
\hline
\end{tabular}

Tabela 3: Distribuição das farmácias hospitalares avaliadas por patamar, segundo a SEFH.

\begin{tabular}{ccc}
\hline Nível & $\%$ & Média dos pontos \\
\hline I & 0 & 0 \\
II & 0 & 0 \\
III & 16,7 & 49,3 \\
IV & 83,3 & 17,4 \\
\hline
\end{tabular}

Dos 12 hospitais avaliados pelo guia proposto, apenas 3 obtiveram pontuação inferior a 50, sendo a pontuação mínima equivalente a 33,8 e a máxima equivalente a 97,5 (Tabela 4).

Tabela 4: Pontuação final obtida pelos hospitais no guia proposto.

\begin{tabular}{cc}
\hline Hospital & Pontuação obtida \\
\hline PPP1 & 33,8 \\
PPP2 & 36,8 \\
PMP1 & 43,9 \\
PMP2 & 56,2 \\
PMP3 & 97,5 \\
PGP1 & 78,8 \\
PUPP1 & 51,8 \\
PUPP2 & 51,4 \\
PUPP3 & 53,4 \\
PUMP1 & 54,7 \\
PUGP1 & 70,0 \\
PUGP2 & 62,5 \\
Média de pontos & 57,6 \\
\hline
\end{tabular}

O guia espanhol classifica as farmácias hospitalares avaliadas com níveis de qualidade em patamares inferiores, indicando maior rigor na qualificação dos serviços, já o guia proposto classifica os serviços com melhor padrão e qualidade, como se pode notar pelas pontuações obtidas. As diferenças das farmácias hospitalares avaliadas com as farmácias hospitalares espanholas podem explicar a pontuação obtida no guia espanhol.

Entre estas diferenças destaca-se a falta do profissional farmacêutico, segundo Osório-de-Castro (2004), cerca de $25 \%$ das farmácias hospitalares no Brasil não possuem farmacêutico. Os dados obtidos revelam que na amostra pesquisada, dois hospitais (16,7\%) não têm farmacêutico responsável, sendo que a ausência do profissional foi exclusiva dos hospitais privados de pequeno porte (PPP). Estes hospitais foram os que obtiveram menores pontuações nas duas avaliações, indicando que a qualidade dos serviços de farmácia hospitalar pode estar relacionada a presença do farmacêutico.

Os dois hospitais que não possuem o profissional farmacêutico contam com a assistência dos farmacêuticos da Associação dos Hospitais do Estado de Goiás (AHEG). Devido ao reduzido número de farmacêuticos da AHEG, apenas 3, e o grande número de hospitais associados atendidos, os profissionais não conseguem prestar uma assistência adequada às instituições, pois só "visitam" os hospitais uma vez por semana para realizar basicamente a escrituração do livro de medicamentos sujeitos a controle especial. Todas as outras 
Martins, B. P. R. et al./Revista Eletrônica de Farmácia Vol 5(1), 83-94, 2008.

atividades inerentes à farmácia hospitalar ficam sob a responsabilidade de outro profissional que não possui formação adequada.

A Tabela 5 mostra a média de pontos obtida quando se leva em conta o regime jurídico da instituição. Observa-se que não houve variação significativa no guia proposto, cujos valores foram de 57,8 e 57,3, para privado e público, respectivamente. Já o guia da SEFH, classifica as farmácias dos hospitais privados como melhores do que as dos públicos, tendo como médias 26,7 e 18,7, respectivamente.

O desvio padrão dos pontos gerais das amostras para os hospitais privados foi de 25,4 e 18,3, segundo os guias proposto e da SEFH, respectivamente. Para os hospitais públicos foi de 7,4 e 5,8 segundo os guias proposto e da SEFH, respectivamente. De acordo com os dois guias, os hospitais públicos têm características mais semelhantes entre si do que os hospitais privados.

Tabela 5: Média de pontos obtida pelas farmácias hospitalares em cada guia de avaliação, por regime jurídico da instituição

\begin{tabular}{ccccc}
\hline & \multicolumn{2}{c}{ Guia SEFH } & \multicolumn{2}{c}{ Guia Proposto } \\
\hline & Público & Privado & Público & Privado \\
\hline Média & 18,7 & 26,7 & 57,3 & 57,8 \\
Desvio padrão & 5,8 & 18,3 & 7,4 & 25,4 \\
\hline
\end{tabular}

A SBRAFH estabelece os padrões mínimos para farmácia hospitalar e as suas atribuições essenciais: gestão, desenvolvimento de infra-estrutura, preparação, distribuição, dispensação e controle de medicamentos e correlatos, informação de medicamentos, promoção do uso racional de medicamentos, ensino e pesquisa (SBRAFH, 2007). Baseado nesta referência selecionou-se as atividades: guia de farmacoterapêutica, consenso terapêutico, comissão de farmácia e terapêutica, sistemas de informação, distribuição por dose unitária e farmacovigilância para avaliar, de acordo com o guia da SEFH, a implantação, atividade e qualidade destas principais atribuições do serviço de farmácia hospitalar, ou seja, avaliar se a atribuição ou atividade está: estabelecida e inserida no serviço, funcionando e se possui excelência/qualidade (Tabela 6).

Tabela 6: Avaliação das principais atividades das farmácias hospitalares, segundo o guia da SEFH

\begin{tabular}{|c|c|c|c|c|c|c|c|}
\hline Atividade & PPP & PMP & PGP & PUPP & PUMP & PUGP & $\begin{array}{c}\text { TOTAL } \\
(\%)\end{array}$ \\
\hline \multicolumn{8}{|c|}{ Guia de farmacoterapêutica } \\
\hline Implantação & 0 & 2 & 1 & 3 & 1 & 2 & 75 \\
\hline Atividade & 0 & 1 & 1 & 0 & 0 & 1 & 25 \\
\hline Qualidade & 0 & 1 & 1 & 0 & 0 & 1 & 25 \\
\hline \multicolumn{8}{|c|}{ Consenso terapêutico } \\
\hline Implantação & 0 & 1 & 1 & 0 & 0 & 0 & 16,7 \\
\hline Atividade & 0 & 1 & 1 & 0 & 0 & 0 & 16,7 \\
\hline Qualidade & 0 & 1 & 1 & 0 & 0 & 0 & 16,7 \\
\hline \multicolumn{8}{|c|}{ Comissão de farmácia e terapêutica } \\
\hline Implantação & 0 & 1 & 1 & 0 & 0 & 1 & 25 \\
\hline Atividade & 0 & 1 & 1 & 0 & 0 & 1 & 25 \\
\hline Qualidade & 0 & 0 & 1 & 0 & 0 & 1 & 16,7 \\
\hline \multicolumn{8}{|c|}{ Sistemas de informação } \\
\hline Implantação & 1 & 3 & 1 & 2 & 1 & 2 & 83,3 \\
\hline Atividade & 1 & 1 & 1 & 2 & 0 & 1 & 50 \\
\hline Qualidade & 1 & 3 & 0 & 1 & 0 & 1 & 50 \\
\hline
\end{tabular}

Distribuição por dose unitária

$\begin{array}{lllll}0 & 0 & 0 & 0 & 8,3\end{array}$ 
Martins, B. P. R. et al./Revista Eletrônica de Farmácia Vol 5(1), 83-94, 2008.

$\begin{array}{llllllll}\text { Atividade } & 0 & 1 & 0 & 0 & 0 & 0 & 8,3 \\ \text { Qualidade } & 0 & 1 & 0 & 0 & 0 & 0 & 8,3\end{array}$

Farmacovigilância

\begin{tabular}{cccccccc} 
Implantação & 0 & 0 & 0 & 0 & 0 & 1 & 8,3 \\
Atividade & 0 & 0 & 0 & 0 & 0 & 1 & 8,3 \\
Qualidade & 0 & 0 & 0 & 0 & 0 & 0 & 0 \\
\hline
\end{tabular}

Os resultados encontrados revelam que a maioria dos serviços possui o guia de farmacoterapêutica, também denominado seleção de medicamentos, implantados (75\%), mas poucos em atividade (25\%) e com qualidade (25\%). A seleção de medicamentos constitui uma das atividades básicas realizadas pela farmácia hospitalar. A partir da sua elaboração são estabelecidos métodos seguros e eficazes para a distribuição e informação dos medicamentos, para realizar essa atividade se faz necessário constituir uma comissão de farmácia e terapêutica (AQUILAR \& BITTNER, 1997). O guia de farmacoterapêutica pode conter apenas a relação dos medicamentos utilizados pelo hospital, como também informações importantes sobre os medicamentos, como via de administração, interações, dosagens etc. Esse guia, principalmente o mais completo, tem um papel importantíssimo no hospital, pois promove a qualidade da prescrição, gestão adequada dos recursos financeiros e o uso seguro de medicamentos (MARQUES \& ZUCCHI, 2006). Todos os hospitais pesquisados possuem apenas a relação de medicamentos.

O consenso terapêutico no hospital constitui a elaboração de protocolos de tratamento por uma equipe multiprofissional, geralmente organizada na forma de comissões intra-hospitalares, essa atividade contribui muito para a assistência ao paciente, pois preconiza o uso seguro e correto dos medicamentos e intervenções.

Diversos estudos revelam que o trabalho de uma equipe multiprofissional é essencial para alcançar os objetivos clínicos e terapêuticos, e de acordo com a OPAS e Ministério da Saúde é competência do farmacêutico hospitalar participar de comissões internas (PENAFORTE, FORSTER \& SILVA, 2007).

O consenso terapêutico está implantado, em atividade e com qualidade em 16,67\% dos hospitais pesquisados, no entanto, essa atividade funciona sem a atuação efetiva do profissional farmacêutico.

A comissão de farmácia e terapêutica (CFT) é constituída por uma equipe multiprofissional e suas principais funções são: seleção, programação, dispensação, elaboração de protocolos terapêuticos e uso racional de medicamentos (AGUILAR \& BITTNER, 1997; WILKEN, 1998). O objetivo principal da comissão é possibilitar que os pacientes recebam o melhor e mais efetivo tratamento. No Brasil a existência da CFT é recomendada, mas não exigida (MARQUES \& ZUCCHI, 2006). Esta atividade está implantada e em atividade em 25\% dos hospitais, mas apenas $16,67 \%$ possuem qualidade.

A gestão adequada de medicamentos e materiais é o pilar da farmácia hospitalar e deve ser realizada por um eficaz sistema de informação, que forneça dados sobre aquisição, consumo, rotatividade do estoque, dados dos produtos que mantém a rastreabilidade do processo como lote, validade entre outras informações. Os sistemas de informação estão implantados em $83,33 \%$ dos hospitais pesquisados, $50 \%$ em atividade e $50 \%$ com qualidade.

Um sistema de dispensação e distribuição de medicamentos seguro, efetivo e organizado são fundamentais para assegurar que os medicamentos sejam dispensados de acordo com a prescrição médica com reduzida possibilidade de erros.

O sistema de distribuição por dose unitária é o mais seguro dos sistemas, diminui as taxas de erros de medicação de 1 erro/paciente/dia para 2 a 3 erros/paciente/semana (ANACLETO et al., 2007). As recomendações da American Society of Hospital Pharmacists - ASHP, para evitar erros de medicação são: uso do código de barras, sistema de monitoramento de eventos adversos, distribuição por dose unitária, sistema de informação de medicamentos e atuação conjunta de todos os profissionais envolvidos com o medicamento (ASHP apud CASSIANI et al., 2005).

Nos hospitais pesquisados, apenas um (8,33\%) possui este sistema implantado, em atividade e com qualidade. A maior parte dos hospitais possui o sistema de distribuição misto e individualizado, facilitando assim a ocorrência de erros de medicação e aumento de eventos adversos. O sistema de distribuição por dose unitária é inicialmente oneroso o que torna mais difícil sua implantação nos hospitais goianos, em especial os públicos que sofrem pela má distribuição de recursos da saúde.

A farmacovigilância está implantada e em atividade em apenas um hospital (8,33\%), mas este serviço não possui qualidade.

Estes resultados revelam que faltam investimentos no serviço de farmácia, principalmente para custear atividades essenciais que podem beneficiar a instituição, com a redução de gastos, e beneficiar os pacientes, que receberão assistência segura, correta e de qualidade.

Os dados apresentados a seguir foram identificados, pelo guia proposto, nos hospitais pesquisados. 
Martins, B. P. R. et al./Revista Eletrônica de Farmácia Vol 5(1), 83-94, 2008.

Tabela 7: Porcentagem do tipo de atendimento realizado e média de leitos dos hospitais.

\begin{tabular}{llll}
\hline & PEQUENO & MÉDIO & GRANDE \\
\hline Atendimento especializado & $100 \%$ & $75 \%$ & $0 \%$ \\
Média de leitos por hospital & 29,6 & 120 & 268 \\
\hline
\end{tabular}

Tabela 8: Horário de funcionamento, farmacêutico responsável e quantidade média de farmacêuticos e | funcionários, de acordo com o porte do hospital.

\begin{tabular}{llll}
\hline & Pequeno & Médio & Grande \\
\hline Serviço de farmácia que funciona $24 \mathrm{~h}$ & $20 \%$ & $50 \%$ & $100 \%$ \\
Farmacêutico responsável & $60 \%$ & $100 \%$ & $100 \%$ \\
Quantidade média de farmacêuticos & 1,7 & 2,7 & 8,0 \\
Quantidade média de funcionários & 3,8 & 13,2 & 17,0 \\
\hline
\end{tabular}

O setor de compras de medicamentos é independente da farmácia em todos os hospitais, exceto nos hospitais de pequeno porte, onde $20 \%$ a farmácia é responsável pelas compras. O estoque disponível é a informação utilizada para realização das compras em 100\% dos hospitais de grande porte, 50\% dos médios e em $80 \%$ dos pequenos. A utilização da curva ABC para programação do estoque é feita somente por $68 \%$ dos hospitais de grande porte. Todos os grandes e médios portes e $80 \%$ dos pequenos possuem cadastro de fornecedores. Em $100 \%$ dos grandes e $75 \%$ dos médios a farmácia fornece parecer técnico para o processo de compra.

Todos os hospitais pesquisados possuem central de abastecimento farmacêutico, onde são armazenados medicamentos e correlatos. Em 100\% dos grandes, $75 \%$ dos médios e $40 \%$ dos pequenos as boas práticas de estocagem são seguidas. Em 100\% dos grandes e 75\% dos médios existem farmácias satélites. Em 100\% dos grandes e médios e em $80 \%$ dos pequenos o controle de estoque é realizado por um programa informatizado.

Em todos os hospitais de grande e médio porte e em $40 \%$ dos hospitais de pequeno porte há participação do farmacêutico na Comissão de Controle de Infecção Hospitalar $(\mathrm{CCIH})$. Nenhum hospital de grande porte executa o Plano de Gerenciamento de Resíduos de Serviços de Saúde (PGRSS), somente 75\% dos médios e $40 \%$ dos pequenos o realizam.

O sistema de distribuição adotado pelos hospitais de grande porte é misto (coletivo e individualizado), sendo exclusivamente individualizado em $34 \%$ dos grandes, $50 \%$ dos médios e $60 \%$ dos pequenos. Apenas um hospital de médio porte possui o sistema de distribuição por dose unitária. Estes dados mostram que o Sistema de distribuição de medicamentos por dose unitária ainda não está consolidado nas farmácias hospitalares goianas.

A dispensação é realizada na presença do farmacêutico em 34\% dos hospitais de grande porte, 50\% dos médios e 60\% dos pequenos. Dos hospitais pesquisados, 68\% dos grandes, 100\% dos médios e $80 \%$ dos pequenos cumprem a Portaria 344/1998 para dispensar medicamentos controlados.

A farmácia participa das pesquisas desenvolvidas nos hospitais em 34\% dos grandes, 25\% dos médios e $20 \%$ dos pequenos. Apenas $24 \%$ dos hospitais de grande porte realizam treinamento e capacitação de seus funcionários frente a $75 \%$ dos médios e $80 \%$ dos pequenos.

Nenhum hospital exerce a farmácia clínica. Nenhum hospital possui um sistema de informações de medicamentos oficial. Os farmacêuticos sempre fornecem informações sobre os medicamentos, mas esta atividade não é registrada nem organizada. Apenas um hospital realiza farmacovigilância, mas não com qualidade, conforme verificado pela avaliação do guia espanhol.

Em síntese as atividades realizadas pela farmácia hospitalar identificadas nos hospitais pesquisados foram: armazenamento e distribuição em $100 \%$ dos hospitais, função administrativa em 83,34\%, dispensação ambulatorial em 41,67\%, manipulação 25\% e controle de qualidade em 8,34\%, como exposto no Gráfico 2. 
Martins, B. P. R. et al./Revista Eletrônica de Farmácia Vol 5(1), 83-94, 2008.

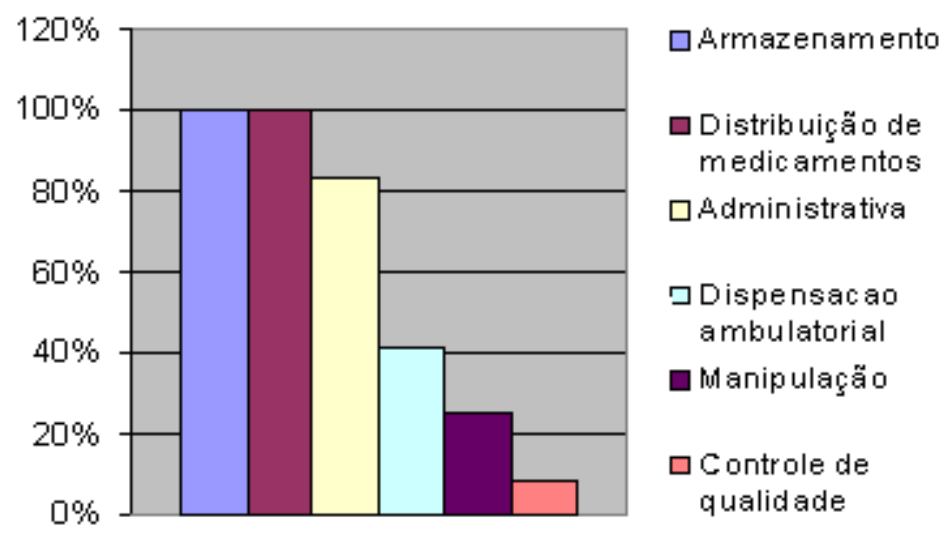

Gráfico 2: Principais atividades realizadas pelos serviços de farmácia hospitalar, nos hospitais pesquisados.

Em comparação ao trabalho "Diagnóstico da farmácia hospitalar no Brasil" (OSÓRIO-DE-CASTRO et al., 2004) conforme exposto na Tabela 9, as farmácias goianas apresentaram, na maioria dos aspectos, melhor qualificação. Esta diferença pode ser devido ao fato do "Diagnóstico da farmácia hospitalar" ter sido realizado em uma amostra representativa de todos os hospitais brasileiros e ter avaliado uma população muito mais heterogênea do que a amostra deste trabalho.

Tabela 9: Comparação entre os dados do trabalho "Diagnóstico da farmácia hospitalar no Brasil" (2004), com os dados obtidos pela avaliação com o guia proposto.

\begin{tabular}{|c|c|c|c|c|c|}
\hline Aspecto & $\begin{array}{l}\text { Pequeno } \\
\text { (\%) }\end{array}$ & $\begin{array}{l}\text { Médio } \\
\text { (\%) }\end{array}$ & $\begin{array}{l}\text { Grande } \\
\text { (\%) }\end{array}$ & Total (\%) & $\begin{array}{c}{\text { Diagnóstico } \mathrm{FH}^{1}}_{(\%)} \\
\text { (\%) }\end{array}$ \\
\hline $\begin{array}{llll}\text { Sistema de } & \text { controle } & \text { de } & \text { estoque } \\
\text { informatizado } & & & \\
\end{array}$ & 80,0 & 100,0 & 100,0 & 91,7 & 55,5 \\
\hline Utilização da curva ABC & 0 & 0 & 68,0 & 25,0 & 30,1 \\
\hline Cadastro de fornecedores & 80,0 & 100,0 & 100,0 & 91,7 & 70,0 \\
\hline $\begin{array}{l}\text { Especificações técnicas elaboradas } \\
\text { pelo farmacêutico }\end{array}$ & 0 & 75,0 & 100,0 & 50,0 & 1,3 \\
\hline Boas práticas de estocagem & 40,0 & 75,0 & 100,0 & 66,7 & 47,6 \\
\hline Sistema de distribuição individualizado & 60,0 & 50,0 & 34,0 & 50,0 & 34,8 \\
\hline $\begin{array}{l}\text { Sistema de distribuição por dose } \\
\text { unitária }\end{array}$ & 0 & 25,0 & 34,0 & 16,7 & 0,4 \\
\hline Farmácia satélite & 0 & 75,0 & 100,0 & 50,0 & 19,6 \\
\hline Participação do farmacêutico na CCIH & 40,0 & 100,0 & 100,0 & 75,0 & 84,2 \\
\hline Existência de uma CFT & 0 & 25,0 & 100,0 & 33,3 & 11,6 \\
\hline Seleção de medicamentos atualizada & 60,0 & 75,0 & 100,0 & 75,0 & 27,2 \\
\hline Formulário terapêutico & 0 & 0 & 0 & 0 & 5,6 \\
\hline Protocolos terapêuticos & 0 & 20,0 & 67,0 & 25,0 & 2,0 \\
\hline $\begin{array}{l}\text { Sistema de informação de } \\
\text { medicamentos }\end{array}$ & 0 & 0 & 0 & 0 & 1,6 \\
\hline Farmácia Clínica & 0 & 0 & 0 & 0 & 0 \\
\hline Farmacovigilância & 0 & 0 & 0 & 0 & 0,4 \\
\hline Treinamento e capacitação & 80,0 & 75,0 & 24,0 & 66,7 & 19,2 \\
\hline
\end{tabular}

${ }^{1} \mathrm{FH}$ : farmácia hospitalar

Outro trabalho, publicado em 2007 avaliou e hierarquizou as farmácias hospitalares no Brasil, de acordo com o desempenho e complexidade dos serviços. Nenhum serviço foi classificado como bom, todos os hospitais estão na categoria insuficiente. Dos hospitais analisados os melhores serviços são dos hospitais públicos, filantrópicos e universitários apresentando maior adequação na qualidade dos serviços quando comparados com hospitais privados (MESSEDER, OSÓRIO-DE-CASTRO \& CAMACHO, 2007).

Goiânia possui uma população estimada de 1.220 .412 habitantes (IBGE, 2007) e grande parte dos seus hospitais não possuem um serviço de farmácia hospitalar com funções e atividades definidas, confiável, nem de qualidade. O serviço de farmácia hospitalar em Goiânia não é compreendido por sua importância clínica e 
Martins, B. P. R. et al./Revista Eletrônica de Farmácia Vol 5(1), 83-94, 2008.

administrativa e sim como um setor do hospital que apenas distribui os medicamentos e materiais necessários para o atendimento ao paciente.

Observa-se que o investimento financeiro para a melhora da estrutura física e de equipamentos é mínimo, sendo que existência de instalações apropriadas, equipamentos e demais recursos que garantam o bom desempenho operacional da equipe é imprescindível.

Percebe-se que os resultados encontrados são semelhantes aos encontrados por Messeder et. al (2007) e Osório-de-Castro et. al. (2004) e revelam que os serviços avaliados precisam urgentemente de uma reestruturação e qualificação.

No sentido de reestruturar e melhorar os serviços de farmácia, várias medidas deveriam ser tomadas iniciando-se na atualização dos currículos dos cursos de graduação, com implementação de disciplinas de atenção farmacêutica e farmácia clínica; elaboração de cursos de educação continuada para os profissionais e união dos órgãos regulamentadores com aprimoramento da fiscalização nestes serviços. Também é necessário, juntamente com, melhores políticas de saúde e de medicamentos, a inserção do profissional farmacêutico nas equipes multiprofissionais de saúde. Na reestruturação institucional, destaca-se a necessidade de reavaliação das rotinas de trabalho, eliminação da sobrecarga administrativa, desburocratização do serviço e reorganização das funções do farmacêutico.

\section{CONCLUSÃo}

Conclui-se que os dois guias de avaliação são úteis, no entanto, o guia proposto pela SEFH se mostrou mais sensível, seus resultados fornecem informações aprofundadas sobre os serviços de farmácia hospitalar, porém, inadequado na rotina brasileira de avaliação.

Esforços futuros devem ser mobilizados no sentido de elaborar, testar e validar novos instrumentos para a avaliação dos serviços de farmácias hospitalares adaptados a realidade brasileira, com o objetivo de constante aperfeiçoamento do setor, bem como melhorar a qualidade da assistência ao paciente.

\section{REFERÊNCIAS BIBLIOGRÁFICAS}

AGUILAR, N.G.; BITTNER, M.R. Seleccíon y Formulario de Medicamentos. Guia para el Desarrolo de Servicios Farmacêuticos Hospitalarios. Serie medicamentos esenciales y tecnologia, número 5.1; 1997.

ANACLETO, T.A. et al. Erros de dispensação de medicamentos em farmácia hospitalar. Revista Clinics. São Paulo; 2007.

BRASIL. Conselho Federal de Farmácia. Resolução no 300 de 30 de janeiro de 1997a. Regulamenta o exercício profissional em farmácia de unidade hospitalar, clinicas e unidades de saúde, de natureza publica ou privada.

BRASIL. Agência Nacional de Vigilância Sanitária. Resolução no 921 de 29 de maio de 2002b. Reconhece a Organização Nacional de Acreditação - ONA. Disponível no site www.anvisa.gov.br/legislaçao, acessado em $23 / 11 / 2007$ as $16: 45$.

CASSIANI, S.H.D.B. et al. O sistema de medicação nos hospitais e sua avaliação por um grupo de profissionais. Revista Esc. Enfermagem. São Paulo; 2005.

CASTRO, I. et al. Documentacíon y evaluacíon de las intervenciones farmacêuticas. Terceira formación continuada en farmacia hospitalaria. Madrid: Ediciones Mayo; 2000.

DONABEDIAN, A. The quality of care. How can it be assessed? JAMA Vol. 260, N.12, 1988.

GOMES, M.J.V.M.; REIS, A.M.M. Ciências Farmacêuticas: uma abordagem em farmácia hospitalar. São Paulo: Editora Atheneu; 2003.

Instituto Brasileiro de Geografia e Estatística - IBGE. Contagem da população 2007. Disponível no site www.ibge.gov.br/estatistica/populacao/contagem2007, acessado em 12/09/2007 as 11:00.

MAGARINOS-TORRES R.; OSORIO-DE-CASTRO C.G.S.; PEPE V.L.E. Atividades da farmácia hospitalar brasileira para com pacientes hospitalizados: uma revisão da literatura. Ciência Saúde Coletiva. Rio de Janeiro; 2007. 
Martins, B. P. R. et al./Revista Eletrônica de Farmácia Vol 5(1), 83-94, 2008.

MARQUES, D.C.; ZUCCHI, P. Comissões farmacoterapêuticas no Brasil: aquém das diretrizes internacionais. Revista Panamericana de Saúde, Washington; 2006.

MESSEDER, A.M.; OSÓRIO-DE-CASTRO, C.G.S; CAMACHO, L.A.B. Projeto Diagnóstico da Farmácia Hospitalar no Brasil: uma proposta de hierarquização dos serviços. Caderno de Saúde Pública. Rio de Janeiro; 2007.

Organização Nacional de Acreditação - ONA. Conheça a ONA. Disponível no site: www.ona.org.br, acessado em $16 / 10 / 2007$ as 10:50.

OSÓRIO-DE-CASTRO, C.G.S.; CASTILHO, S.R. Organizadores. Diagnóstico da Farmácia Hospitalar no Brasil. Rio de Janeiro: Editora Fiocruz; 2004.

PENAFORTE T.R.; FORSTER A.C.; SILVA M.J.S. Avaliação da Atuação dos Farmacêuticos na Prestação da Assistência a Saúde em um Hospital Universitário. Clinics. São Paulo; 2007.

Sociedade Brasileira de Farmácia Hospitalar - SBRAFH. Padrões Mínimos para Farmácia Hospitalar. São Paulo: SBRAFH; 1996. Disponível no site www.sbrafh.org.br/padrões mínimos para farmácia hospitalar, acessado em $17 / 07 / 2007$ as $12: 30$.

Sociedade Espanhola de Farmacia Hospitalaria - SEFH. Guia de evaluación y mejora de los servicios de farmacia 1 hospitalarios. Disponível no site www.sefh.es/01otras publicaciones.php, acessado em 03/07/2007 as 15:30.

WILKEN, P.R.C. A farmácia no hospital e a atenção a saúde. Assistência farmacêutica nos hospitais do Ministério da Saúde no Rio de Janeiro: estudo de caso. Dissertação de mestrado da Escola Nacional de Saúde Pública da Fundação Oswaldo Cruz. Rio de Janeiro; 1998. 